PHYSICAL REVIEW D 96, 049901(E) (2017)

\title{
Publisher's Note: Rotating (A)dS black holes in bigravity [Phys. Rev. D 93, 024049 (2016)]
}

Eloy Ayón-Beato, Daniel Higuita-Borja, and Julio A. Méndez-Zavaleta

(Received 25 July 2017; published 7 August 2017)

DOI: 10.1103/PhysRevD.96.049901

This paper was published online on 27 January 2016 with an error in the affiliations for the first author. Eloy Ayón-Beato's affiliations should read as

${ }^{1}$ Departamento de Física, CINVESTAV-IPN, Apdo. Postal 14-740, 07000 México D.F., México

${ }^{2}$ Instituto de Ciencias Físicas y Matemáticas, Universidad Austral de Chile, Casilla 567 Valdivia, Chile.

The author's affiliations have been corrected as of 28 July 2017. The author's affiliations are incorrect in the printed version of the journal. 